\title{
Quasi-Cartesian finite-difference computation of seismic wave propagation for a three-dimensional sub-global model
}

\author{
Hiroshi Takenaka ${ }^{1 *}$, Masanao Komatsu' $^{1}$, Genti Toyokuni ${ }^{2}$, Takeshi Nakamura ${ }^{3}$ and Taro Okamoto ${ }^{4}$
}

\begin{abstract}
A simple and efficient finite-difference scheme is developed to calculate seismic wave propagation in a partial spherical shell model of a three-dimensionally (3-D) heterogeneous global Earth structure for modeling on regional or sub-global scales where the effects of the Earth's spherical geometry cannot be ignored. This scheme solves the elastodynamic equation in the quasi-Cartesian coordinate form similar to the local Cartesian one, instead of the spherical polar coordinate form, with a staggered-grid finite-difference method in time domain (FDTD) that is one of the most popular numerical methods in seismic-motion simulations for local-scale models. The proposed scheme may be a local-friendly approach for modeling on a sub-global scale to link regional-scale and local-scale simulations. It can be easily implemented using an available 3-D Cartesian FDTD local-scale modeling code by changing a very small part of the code. We implement the scheme in an existing Cartesian FDTD code and demonstrate the accuracy and validity of the present scheme and the feasibility to apply it to real large simulations through numerical examples.
\end{abstract}

Keywords: Finite-difference method, FDTD, Seismic wave propagation, Sub-global model, Regional scale, Quasi-Cartesian coordinates

\section{Introduction}

In recent years, there have been remarkable developments in numerical modeling techniques of seismic wave propagation, associated with progress in computer architecture. The numerical simulation has become a dominant tool for understanding seismic events in both earthquake seismology and exploration seismology. There are several numerical methods used for such purposes, such as the finite-element, spectral-element, and finite-difference methods. Among these the finitedifference method (FDM) is one of the most popular ones. The FDM can be applied in either the time or frequency domain. The finite-difference method in time domain (FDTD) is popular because it is relatively simple and easy to program. For a review on various schemes of the FDTD, see, e.g., Moczo et al. (2014). Simplicity of the

\footnotetext{
*Correspondence: htakenaka@cc.okayama-u.ac.jp

1 Department of Earth Sciences, Okayama University, 3-1-1

Tsushima-Naka, Kita-ku, Okayama 700-8530, Japan

Full list of author information is available at the end of the article
}

FDTD implementation motivates seismologists to apply this method.

The FDTD has been widely utilized for three-dimensional (3-D) seismic wave simulations on local scales (e.g., Graves 1996; Hayashida et al. 1999; Pitarka 1999). The FDTD has also been successfully applied to globalscale modeling (e.g., Thomas et al. 2000; Toyokuni et al. 2005; Toyokuni and Takenaka 2006). The global-scale modeling usually solves the elastodynamic equation in spherical polar coordinates, while the local-scale modeling solves the equation in Cartesian coordinates. We often deal with a problem of an intermediate scale, so we may search for a simple but accurate or stable scheme. For modeling on regional scales, we may have to consider the spherical geometry of the Earth. Historically, Earth-flattening transformation has been used in waveform modelings for laterally homogeneous (i.e., spherically symmetric) Earth models (see, e.g., Box 9.2 of Aki and Richards 2002) with, for example, the reflectivity method. It can exactly transform a SH-wave propagation problem posed for a medium with spherical symmetry 
into a problem posed for a plane-stratified medium and give a useful approximation to a P-SV problem in a spherically symmetric medium. However, for laterally or three-dimensionally heterogeneous Earth models the validation of the use of Earth-flattening approximation should be eventually checked by comparing the solutions with those computed by numerical methods such as the FDTD without the approximation for each of the same models. The FDTD has actually been applied to modeling wave propagation even for media with random properties (e.g., Igel and Gudmundsson 1997). We thus prefer to use a method without Earth-flattening approximation. The FDTD has been exploited for intermediate-scale, regional- or continental-scale modeling as well as localscale modeling. Igel et al. (2002) presented an excellent scheme for a partial spherical shell (spherical section) model of a 3-D heterogeneous global earth structure, which is a rather global-friendly method to link regionalscale and global-scale modelings because it solves the elastodynamic equation in spherical polar coordinates.

In this paper, we propose a local-friendly approach for modeling on sub-global scales to link regional-scale and local-scale modelings. This solves the elastodynamic equation in the quasi-Cartesian coordinate form similar to local Cartesian one, instead of in the original spherical polar coordinate form, with the FDTD. The proposed scheme can be easily implemented in an available 3-D Cartesian FDTD code of local-scale modeling such as strong-motion simulation by changing a very small part of the code. It is one of the most important merits of use of the quasi-Cartesian formulations instead of the original spherical polar ones.

The quasi-Cartesian approach could be easily applied to a multiscale hybrid method or domain decomposition method that divides the computational domain into multiple domains in which wave propagation is calculated separately by changing grid size and type (e.g., uniform or non-uniform), often with different methods (e.g., Moczo et al. 1997; Wen and Helmberger 1998; Robertsson and Chapman 2000; Yoshimura et al. 2003; Opršal et al. 2009; Monteiller et al. 2013). If we consider a local domain embedded in a sub-global domain (Fig. 1a, c), the wave propagation in the sub-global domain is computed with the quasi-Cartesian FDTD as a background model. In the local domain, the propagation is then calculated with the Cartesian FDTD. In this process, we may be able to couple the computations between the domains through the hybrid of the Cartesian and quasi-Cartesian formulations without combining different methods. For coupling the wavefields in the two domains, for instance, so-called a finite-difference injection method could be exploited, which allows us to calculate synthetic seismograms

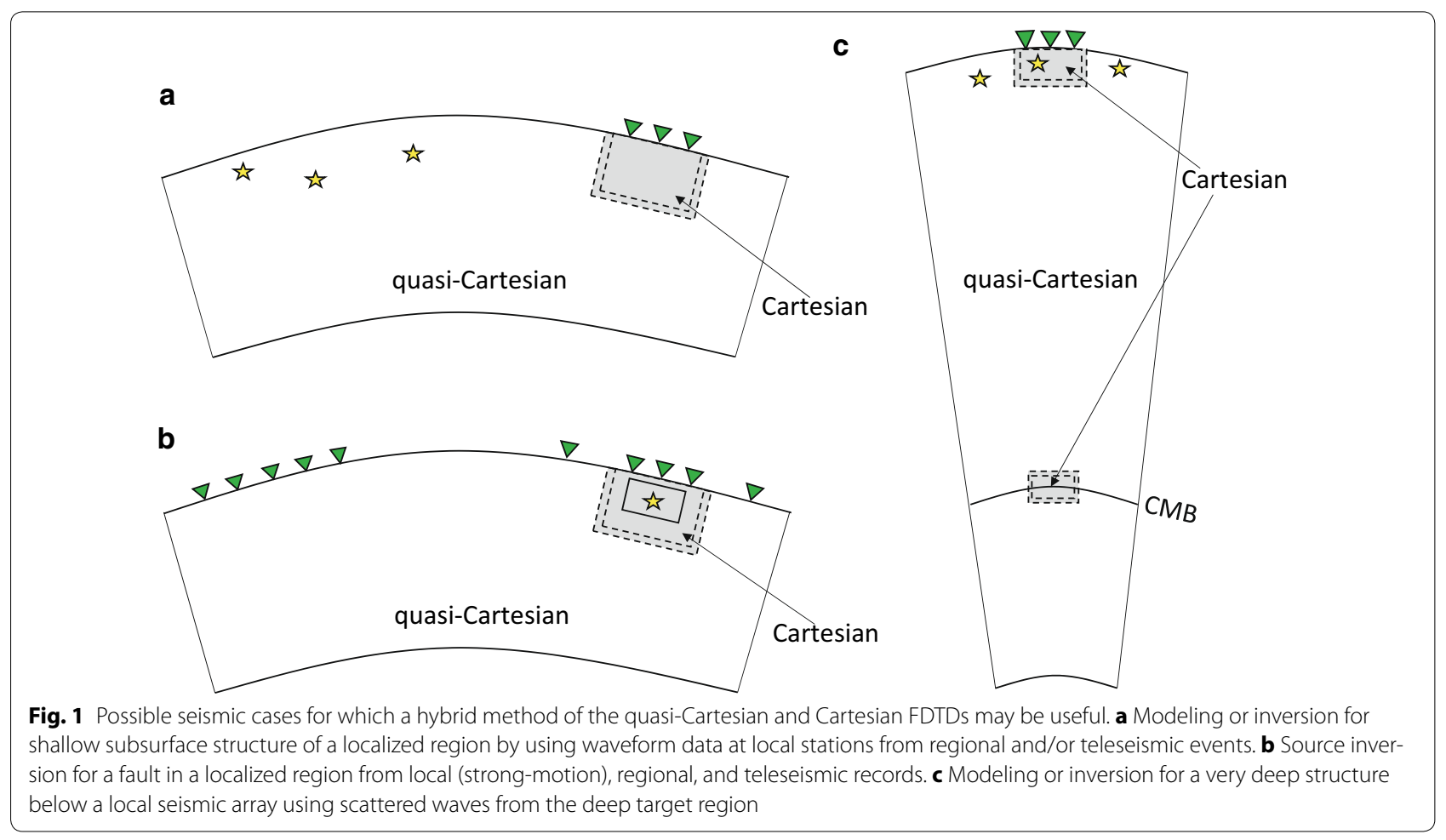


efficiently after model alterations in the local domain (Robertsson and Chapman 2000; Borisov et al. 2015). The hybrid may also be applicable to a source inversion that estimates a spatiotemporal slip distribution on a fault plane from local (strong-motion), regional, and teleseismic records (Fig. 1b). In this case, the target fault may be set in the local domain where Cartesian coordinates are used. These issues on the hybrid method might be nearfuture subjects.

\section{Methods}

For simplicity, we here consider formulations for elastic waves without anelastic attenuation to explain the scheme. In a spherical polar coordinate system $(r, \theta, \phi)$ (Fig. 2), the velocity-stress form of the 3-D isotropic linear elastodynamic equation may be written as (e.g., Igel et al. 2002; Toyokuni et al. 2012):

(equation of motion)

$$
\begin{aligned}
\rho \frac{\partial v_{r}}{\partial t}= & f_{r}+\frac{\partial \sigma_{r r}}{\partial r}+\frac{1}{r} \frac{\partial \sigma_{r \theta}}{\partial \theta}+\frac{1}{r \sin \theta} \frac{\partial \sigma_{r \phi}}{\partial \phi} \\
& +\frac{1}{r}\left(2 \sigma_{r r}-\sigma_{\theta \theta}-\sigma_{\phi \phi}\right)+\frac{1}{r} \sigma_{r \theta} \cot \theta \\
\rho \frac{\partial v_{\theta}}{\partial t}= & f_{\theta}+\frac{\partial \sigma_{r \theta}}{\partial r}+\frac{1}{r} \frac{\partial \sigma_{\theta \theta}}{\partial \theta}+\frac{1}{r \sin \theta} \frac{\partial \sigma_{\theta \phi}}{\partial \phi} \\
& +\frac{3}{r} \sigma_{r \theta}+\frac{1}{r}\left(\sigma_{\theta \theta}-\sigma_{\phi \phi}\right) \cot \theta \\
\rho \frac{\partial v_{\phi}}{\partial t}= & f_{\phi}+\frac{\partial \sigma_{r \phi}}{\partial r}+\frac{1}{r} \frac{\partial \sigma_{\theta \phi}}{\partial \theta}+\frac{1}{r \sin \theta} \frac{\partial \sigma_{\phi \phi}}{\partial \phi} \\
& +\frac{3}{r} \sigma_{r \phi}+\frac{2}{r} \sigma_{\theta \phi} \cot \theta,
\end{aligned}
$$

and (constitutive equation)

$$
\begin{aligned}
\frac{\partial \sigma_{r r}}{\partial t}= & (\lambda+2 \mu) \frac{\partial v_{r}}{\partial r}+\lambda \frac{1}{r} \frac{\partial v_{\theta}}{\partial \theta}+\lambda \frac{1}{r \sin \theta} \frac{\partial v_{\phi}}{\partial \phi} \\
& +2 \lambda \frac{v_{r}}{r}+\lambda \frac{1}{r} v_{\theta} \cot \theta-\dot{M}_{r r} \\
\frac{\partial \sigma_{\theta \theta}}{\partial t}= & \lambda \frac{\partial v_{r}}{\partial r}+(\lambda+2 \mu) \frac{1}{r} \frac{\partial v_{\theta}}{\partial \theta}+\lambda \frac{1}{r \sin \theta} \frac{\partial v_{\phi}}{\partial \phi} \\
& +2(\lambda+\mu) \frac{v_{r}}{r}+\lambda \frac{1}{r} v_{\theta} \cot \theta-\dot{M}_{\theta \theta}, \\
\frac{\partial \sigma_{\phi \phi}}{\partial t}= & \lambda \frac{\partial v_{r}}{\partial r}+\lambda \frac{1}{r} \frac{\partial v_{\theta}}{\partial \theta}+(\lambda+2 \mu) \frac{1}{r \sin \theta} \frac{\partial v_{\phi}}{\partial \phi} \\
& +2(\lambda+\mu) \frac{v_{r}}{r}+(\lambda+2 \mu) \frac{1}{r} v_{\theta} \cot \theta-\dot{M}_{\phi \phi}, \\
\frac{\partial \sigma_{r \theta}}{\partial t}= & \mu \frac{\partial v_{\theta}}{\partial r}+\mu \frac{1}{r} \frac{\partial v_{r}}{\partial \theta}-\mu \frac{v_{\theta}}{r}-\dot{M}_{r \theta}, \\
\frac{\partial \sigma_{\theta \phi}}{\partial t}= & \mu \frac{1}{r} \frac{\partial v_{\phi}}{\partial \theta}+\mu \frac{1}{r \sin \theta} \frac{\partial v_{\theta}}{\partial \phi}-\mu \frac{1}{r} v_{\phi} \cot \theta-\dot{M}_{\theta \phi}, \\
\frac{\partial \sigma_{r \phi}}{\partial t}= & \mu \frac{\partial v_{\phi}}{\partial r}+\mu \frac{1}{r \sin \theta} \frac{\partial v_{r}}{\partial \phi}-\mu \frac{v_{\phi}}{r}-\dot{M}_{r \phi},
\end{aligned}
$$

where $\rho$ is the density, $v_{r}, v_{\theta}$, and $v_{\phi}$ are the particle velocities, $\sigma_{r r}, \sigma_{\theta \theta}, \sigma_{\phi \phi}, \sigma_{r \theta}, \sigma_{\theta \phi}$, and $\sigma_{r \phi}$ are the stress components, $f_{r}, f_{\theta}$, and $f_{\phi}$ are body-force components, $\lambda$ and $\mu$ are Lamé constants, and $\dot{M}_{r r}, \dot{M}_{\theta \theta}, \dot{M}_{\phi \phi}, \dot{M}_{r \theta}, \dot{M}_{\theta \phi}$, and $\dot{M}_{r \phi}$ are the time derivatives of moment-tensor components.

A differential position or coordinate vector $\mathrm{d} \boldsymbol{r}$ may be written in the spherical polar coordinate system as

$$
\begin{aligned}
\mathrm{d} \boldsymbol{r} & =\hat{\boldsymbol{r}} \mathrm{d} r+\hat{\boldsymbol{\theta}} r \mathrm{~d} \theta+\hat{\boldsymbol{\phi}} r \sin \theta \mathrm{d} \phi \\
& =\hat{\boldsymbol{r}} \mathrm{d} s_{r}+\hat{\boldsymbol{\theta}} \mathrm{d} s_{\theta}+\hat{\boldsymbol{\phi}} \mathrm{d} s_{\phi},
\end{aligned}
$$
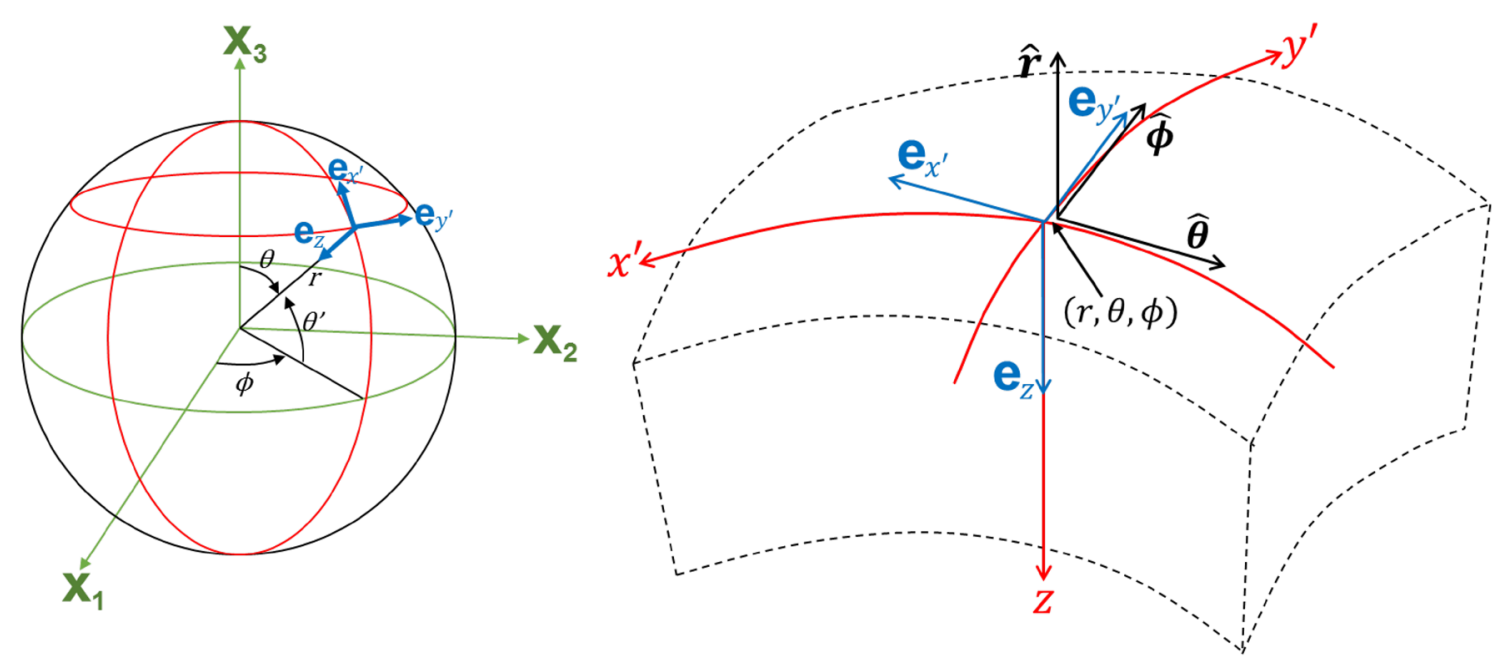

Fig. 2 Relationship between quasi-Cartesian and spherical polar coordinates. Left global view. Right local view 
where $(\hat{\boldsymbol{r}}, \hat{\boldsymbol{\theta}}, \hat{\boldsymbol{\phi}})$ are the spherical polar coordinate unit base vectors (Fig. 2), and $\mathrm{d} s_{r}, \mathrm{~d} s_{\theta}$, and $\mathrm{d} s_{\phi}$ are line elements of the arc lengths $s_{r}, s_{\theta}$, and $s_{\phi}$, respectively.

Changing the coordinate variables as

$$
z=R_{0}-r, \quad \theta^{\prime}=\frac{\pi}{2}-\theta,
$$

where $z$ is depth, $R_{0}$ is the Earth's radius, and $\theta^{\prime}$ is latitude, the differential coordinate vector may be represented as

$$
\mathrm{d} \boldsymbol{r}=\hat{z} \mathrm{~d} s_{z}+\hat{\boldsymbol{\theta}}^{\prime} \mathrm{d} s_{\theta^{\prime}}+\hat{\boldsymbol{\phi}} \mathrm{d} s_{\phi} .
$$

Relabeling $\left(\hat{\boldsymbol{z}}, \hat{\boldsymbol{\theta}}^{\prime}, \hat{\boldsymbol{\phi}}\right), \mathrm{d} s_{z^{\prime}}, \mathrm{d} s_{\theta^{\prime}}$, and $\mathrm{d} s_{\phi}$ as $\left(\mathbf{e}_{z^{\prime}}, \mathbf{e}_{x^{\prime}}, \mathbf{e}_{y^{\prime}}\right), \mathrm{d} z$, $\mathrm{d} x^{\prime}$, and $\mathrm{d} y^{\prime}$, respectively,

$$
\begin{aligned}
& \mathrm{d} \boldsymbol{r}=\mathbf{e}_{z} \mathrm{~d} z+\mathbf{e}_{x^{\prime}} \mathrm{d} x^{\prime}+\mathbf{e}_{y^{\prime}} \mathrm{d} y^{\prime}, \\
& \mathrm{d} x^{\prime}=r \mathrm{~d} \theta^{\prime}, \quad \mathrm{d} y^{\prime}=r \cos \theta^{\prime} \mathrm{d} \phi .
\end{aligned}
$$

Note that the unit vectors $\mathbf{e}_{z^{\prime}}, \mathbf{e}_{x^{\prime}}$, and $\mathbf{e}_{y^{\prime}}$ are pointing downward, north, and east, respectively, in a local Cartesian coordinate system, which vary in direction as the angles $\theta^{\prime}$ and $\phi$. In Fig. 2, $x^{\prime}$ - and $y^{\prime}$-curves are identical to the latitude and longitude lines passing a position $(r$, $\theta, \phi)$, respectively. We here call the coordinates $\left(z, x^{\prime}, y^{\prime}\right)$ quasi-Cartesian coordinates. They all have dimension of length. Equation (7) shows that for lateral derivatives $\partial \bullet / r \partial \theta^{\prime}=\partial \bullet / \partial x^{\prime}$ and $\partial \bullet / r \cos \theta^{\prime} \partial \phi=\partial \bullet / \partial y^{\prime}$.

There are simple relations (one-to-one correspondence) among the components of the particle velocity vector and the stress tensor in the original spherical polar coordinate and the quasi-Cartesian coordinate systems. Using these relations and the differential coordinates in Eqs. (6) and (7), we can get the form of the elastodynamic equation in quasi-Cartesian coordinates:

$$
\begin{aligned}
& \rho \frac{\partial v_{z}}{\partial t}=f_{z}+\frac{\partial \sigma_{z z}}{\partial z}+\frac{\partial \sigma_{z x^{\prime}}}{\partial x^{\prime}}+\frac{\partial \sigma_{z y^{\prime}}}{\partial y^{\prime}} \quad-\frac{1}{r}\left(2 \sigma_{z z}-\sigma_{x^{\prime} x^{\prime}}-\sigma_{y \prime y}\right)-\frac{1}{r} \sigma_{z x^{\prime}} \tan \theta^{\prime}, \text { । } \\
& \rho \frac{\partial v_{x^{\prime}}}{\partial t}=f_{x^{\prime}}+\frac{\partial \sigma_{z x \prime}}{\partial z}+\frac{\partial \sigma_{x^{\prime} x^{\prime}}}{\partial x^{\prime}}+\frac{\partial \sigma_{x^{\prime} y^{\prime}}}{\partial y^{\prime}}:-\frac{3}{r} \sigma_{z x_{\prime}}-\frac{1}{r}\left(\sigma_{x^{\prime} x^{\prime}}-\sigma_{y^{\prime} y^{\prime}}\right) \tan \theta^{\prime}, \\
& \rho \frac{\partial v_{y^{\prime}}}{\partial t}=f_{y^{\prime}}+\frac{\partial \sigma_{z y^{\prime}}}{\partial z}+\frac{\partial \sigma_{x^{\prime} y^{\prime}}}{\partial x^{\prime}}+\frac{\partial \sigma_{y^{\prime} y^{\prime}}}{\partial y^{\prime}}-\frac{3}{r} \sigma_{z y^{\prime}}-\frac{2}{r} \sigma_{x^{\prime} y^{\prime}} \tan \theta^{\prime} \\
& \frac{\partial \sigma_{z z}}{\partial t}=(\lambda+2 \mu) \frac{\partial v_{z}}{\partial z}+\lambda \frac{\partial v_{x \prime}}{\partial x^{\prime}}+\lambda \frac{\partial v_{y \prime}}{\partial y^{\prime}}: 2 \lambda \frac{-\overline{v_{z}}}{r}-\lambda \frac{T}{r} v_{x^{\prime}} \tan \theta^{\prime} \\
& \frac{\partial \sigma_{x \prime x^{\prime}}}{\partial t}=\lambda \frac{\partial v_{z}}{\partial z}+(\lambda+2 \mu) \frac{\partial v_{x \prime}}{\partial x^{\prime}}+\lambda \frac{\partial v_{y \prime}}{\partial y^{\prime}}-2(\lambda+\mu) \frac{v_{z}}{r}-\lambda \frac{1}{r} v_{x^{\prime}} \tan \theta^{\prime} \\
& \frac{\partial \sigma_{y \prime} y^{\prime}}{\partial t}=\lambda \frac{\partial v_{z}}{\partial z}+\lambda \frac{\partial v_{x \prime}}{\partial x^{\prime}}+(\lambda+2 \mu) \frac{\partial v_{y \prime}}{\partial y^{\prime}} \stackrel{I}{-} 2(\lambda+\mu) \frac{v_{z}}{r}-(\lambda+2 \mu) \frac{1}{r} v_{x \prime} \tan \theta^{\prime}{ }_{\text {I }}-\dot{M}_{y^{\prime} y^{\prime}} \text {, } \\
& \frac{\partial \sigma_{z x \prime}}{\partial t}=\mu \frac{\partial v_{x \prime}}{\partial z}+\mu \frac{\partial v_{z}}{\partial x^{\prime}}+\mu \frac{v_{x \prime}}{r} \quad-\dot{M}_{z x \prime}, \\
& \frac{\partial \sigma_{x \prime y^{\prime}}}{\partial t}=\mu \frac{\partial v_{y^{\prime}}}{\partial x^{\prime}}+\mu \frac{\partial v_{x^{\prime}}}{\partial y^{\prime}}+\mu \frac{1}{r} v_{y \prime} \tan \theta^{\prime}-\dot{M}_{x \prime y \prime}, \\
& \frac{\partial \sigma_{y \prime z}}{\partial t}=\mu \frac{\partial v_{y \prime}}{\partial z}+\left.\mu \frac{\partial v_{z}}{\partial y^{\prime}}\right|_{-}+\mu \frac{v_{y \prime}}{r} \quad-\dot{M}_{y \prime z}
\end{aligned}
$$


In derivation of these equations, we have carried out simple variable changes such as Eq. (4) and relabeling some coordinate-related quantities but have not introduced any approximation. The quasi-Cartesian form, a set of Eqs. (8), (9) and (7), is equivalent to the original spherical polar form and is exact. We found that each of quasiCartesian Eqs. (8) and (9) is formally identical to the corresponding 3-D Cartesian coordinate equation except $r$-dependence of $\mathrm{d}^{\prime}, r$ - and $\theta^{\prime}$-dependence of $\mathrm{d} y x^{\prime}$, and the terms with $1 / r$ which are enclosed by blue-dashed lines and called "additional terms" hereafter. This fact means that we could implement a set of Eqs. (8), (9) and (7) by adding the additional terms in an available computer code for 3-D local Cartesian form and using the lateral grid intervals $\Delta x$ and $\Delta y$ depending on the radius $r$ (or depth $z$ ) and the latitude $\theta^{\prime}$ as

$$
\Delta x=r \Delta \theta^{\prime}, \quad \Delta y=r \cos \theta^{\prime} \Delta \phi .
$$

The finite-difference stencil (a set of weights) for each of the $x^{\prime}$-derivatives in the modified code then depends on the depth coordinate at the evaluation point, while the stencil for each of the $y^{\prime}$-derivatives varies with both coordinates of the depth and latitude. Note that the grid points for the FDM of the quasi-Cartesian form are identical to those of the spherical polar form.

In the staggered-grid FDMs such as the FDTD, the derivatives of every field quantity are naturally defined halfway between the grid points where the field quantity is defined. Thus, terms on the right-hand side of the elastodynamic equation [Eqs. (8) and (9)], including spatial derivatives, are consistently evaluated at the same grid position where the field quantity on the left-hand side is defined. However, this is not the case for terms that do not include spatial derivatives, i.e., the additional terms in Eqs. (8) and (9). In our FDTD scheme, these terms are evaluated using Lagrange interpolation of the same accuracy order as the corresponding finite difference (e.g., Fornberg 1988).

This scheme can be easily implemented using an available 3-D Cartesian FDTD code of local-scale modeling. We have implemented the scheme in an existing Cartesian FDTD code (Nakamura et al. 2012) which can calculate local seismic wave propagation in a 3-D land-ocean unified model with sea layer, topography, and anelastic attenuation by the FDTD of fourth-order accuracy in space and second-order accuracy in time. This code utilizes a unified scheme for fluid-solid boundary to correctly model land and seafloor topography (Takenaka et al. 2009) and employs perfectly matched layer (PML) absorbing boundaries to suppress the artificial reflections from the sides and bottom of the computational domain.

Modeling target for the quasi-Cartesian approach may be a limited area of the globe that is a section of a spherical shell. When the simulation area is at higher latitude, the grid spacing of the $y^{\prime}$-direction changes more rapidly. From a point view of the accuracy and the stability of the finite-difference approximation, it is better to keep the variation of spatial grid spacing smaller in the computational domain. A simple approach to do so is to move the target area to around the equator of the computational spherical coordinate system using geometrical rotations of the coordinate axes (e.g., Igel et al. 2002). We exploit the transformation from equatorial coordinates to ecliptic coordinates as one of the methods for this approach, which is widely used in astronomy.

We now describe how to apply the equatorial-toecliptic coordinate transformation to our aim. Before conducting the equatorial-to-ecliptic coordinate transformation, we choose a central position of the target area ("Original" in Fig. 3) as the reference point with latitude of $\varepsilon$ and rotate the computational domain about the north pole so that the longitude of the reference point is

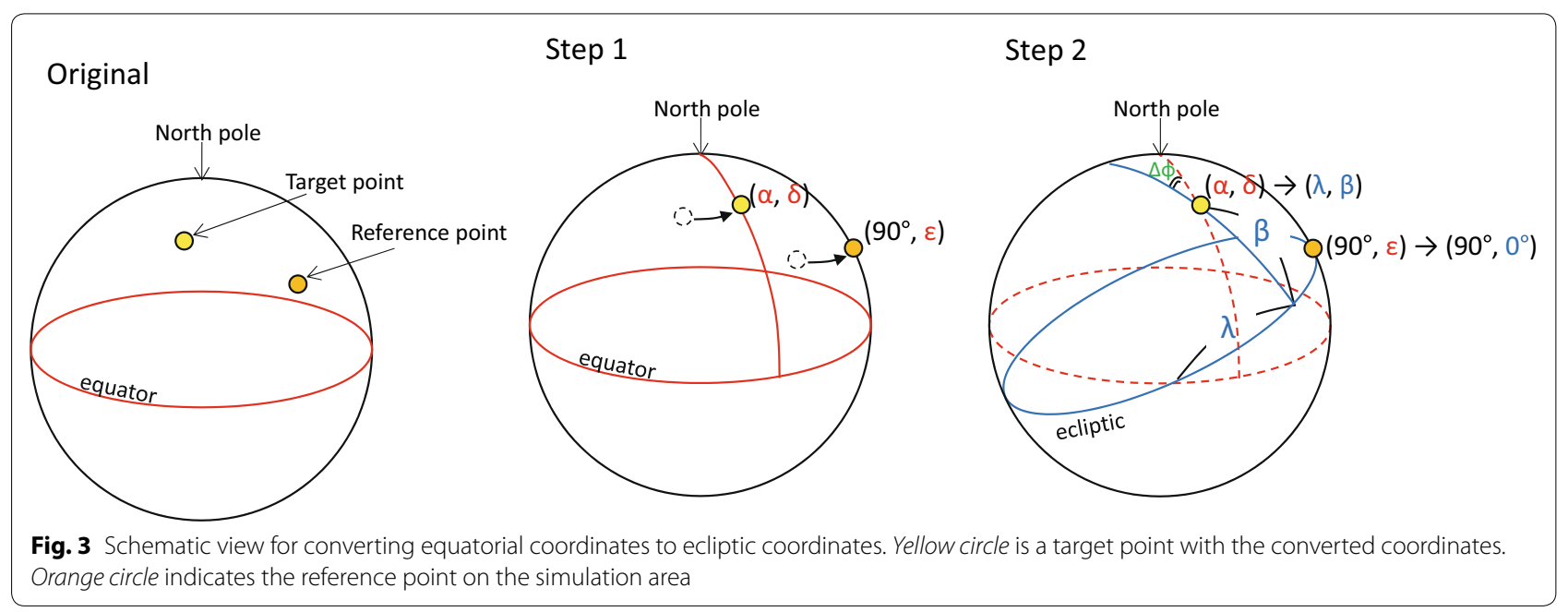


shifted into $90^{\circ} \mathrm{E}$ (“Step 1" in Fig. 3). The equatorial coordinates of the reference point are then (longitude, latitude $)=\left(90^{\circ}, \varepsilon\right)$. We set the ecliptic through the reference point and employ the equatorial-to-ecliptic coordinate transformation which transforms the coordinates of the reference point to $\left(90^{\circ}, 0^{\circ}\right)$ ("Step 2" in Fig. 3). Let us consider an arbitrary point on the computational area with equatorial coordinates $(\alpha, \delta)$, which is indicated by a yellow circle called target point in Fig. 3. Applying the equatorial-to-ecliptic coordinate transformation to the target point gives its ecliptic coordinates $(\lambda, \beta)$ as

$$
\begin{aligned}
& \lambda=\arctan \left(\frac{\sin \delta \sin \varepsilon+\cos \delta \sin \alpha \cos \varepsilon}{\cos \delta \cos \alpha}\right), \\
& \beta=\arcsin (\sin \delta \cos \varepsilon-\cos \delta \sin \alpha \sin \varepsilon) .
\end{aligned}
$$

The angle between the longitude lines through the target point in the equatorial and ecliptic coordinate systems, $\Delta \phi$, is then

$\Delta \phi=\arcsin \left(\frac{\cos \alpha \sin \varepsilon}{\cos \beta}\right)=\arcsin \left(\frac{\cos \lambda \sin \varepsilon}{\cos \delta}\right)$.

This gives the angle change of azimuths in the equatorialto-ecliptic coordinate transformation.

\section{Numerical examples}

In this section, we show two numerical examples of the quasi-Cartesian FDTD application. The first numerical example demonstrates seismic wave propagation in a Moon section model. Since the Moon is smaller than the Earth, the effects of the spherical geometry on wave propagation could be stronger than those of the Earth. We compare waveforms (synthetic seismograms) for a spherical Moon model and the corresponding flat model. The synthetic seismograms for the spherical Moon model are calculated by solving a set of Eqs. (8), (9) and (7) (hereafter called "quasi-Cartesian FDTD"), while those for the flat model are obtained by using the equations without the additional terms and assuming constant $\Delta x$ and $\Delta y$ (hereafter called "Cartesian FDTD"). In the first numerical example, we check the accuracy and validity of the quasi-Cartesian scheme by comparing the synthetic seismograms with those obtained by the spherical FDTD (Toyokuni and Takenaka 2012) which treats an axisymmetric global model including the center of the sphere in spherical polar coordinates. The second numerical example demonstrates a long-range $(\sim 1000 \mathrm{~km})$ simulation of seismic wave propagation around Japan for a virtual subduction event. The structural model for this simulation is three-dimensionally heterogeneous and has land and ocean topographies and seawater. In the second numerical example, we also compare waveforms for a 3-D heterogeneous spherical Earth model with those for the corresponding flat model.

\section{Simulation for a Moon section}

Figure 4 shows the model setting. The computational domain is a spherical cubic section of $45^{\circ} \times 45^{\circ} \times 450 \mathrm{~km}$, which is discretized with cells of $0.06172^{\circ} \times 0.06172^{\circ} \times 1 \mathrm{~km}$ for the quasi-Cartesian FDTD. The spacing of $0.06172^{\circ}$ corresponds to $1.87 \mathrm{~km}$ at the Moon surface. The time step is $0.0125 \mathrm{~s}$. The source (point source) and receivers are located at a depth of $100 \mathrm{~km}$. The datum (acquisition surface) is indicated by light blue surface in Fig. 4. Note that subsurface receivers could be more affected by the spherical geometry than surface receivers because of the shorter radius. The employed seismic structure is spherically symmetric, i.e., 1-D, which is shown in Fig. 5. We constructed

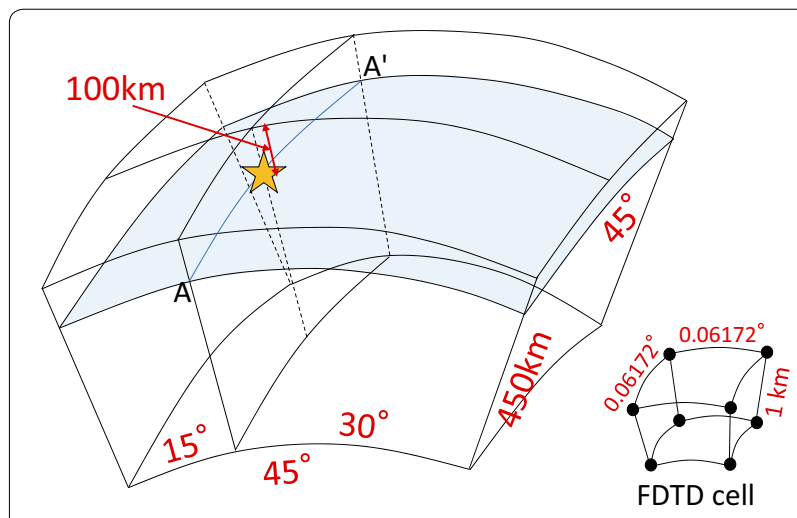

Fig. 4 Model setting for the Moon simulation. Location of the source (star) and the datum (light blue-colored surface). Receivers are set at line $\mathrm{A}-\mathrm{A}^{\prime}$ (blue line) on the datum. This line also indicates location of cross section shown in Fig. 7 for the snapshots. A small curved cubic on the right side of the model schematically shows a grid cell for the computation by the quasi-Cartesian FDTD 




this structure based on Garcia et al. (2011). This model is perfectly elastic without anelastic attenuation. We put an axisymmetric source of $M_{z z}=10^{18} \mathrm{Nm}$ whose source time function is a bell-shaped pulse with width of $15 \mathrm{~s}$.

Figure 6 shows the vertical and radial components of calculated waveforms at seven receivers on $\mathrm{A}-\mathrm{A}^{\prime}$ line shown in Fig. 4. The transverse components are not displayed as they are all zero because of the axisymmetric source. The waveforms are bandpass-filtered in the period range $25-100 \mathrm{~s}$. The traces of the quasi-Cartesian and the spherical FDTDs (spherical models) are almost identical, which illustrates the accuracy and validity of the present quasi-Cartesian scheme. The traces of the Cartesian FDTD (flat model) are different from those of the other FDTDs (spherical models). The discrepancy becomes larger as the epicentral distance increases. Figure 7 shows snapshots of vertical particle velocity field over the vertical cross section including the source and the receivers shown in Fig. 6. It is found that the PML works well for the sides and the bottom of the computational domain.


Fig. 6 Comparison among synthetic seismograms (particle velocity components) by three methods: quasi-Cartesian (red line), spherical (black line) and Cartesian (green line) FDTDs. Right upper and lower panels show the waveforms in the radial and vertical components, respectively. The amplitude of each trace has been multiplied by the epicentral distance. The number beside each trace indicates the receiver number corresponding to the location shown in the left panel 


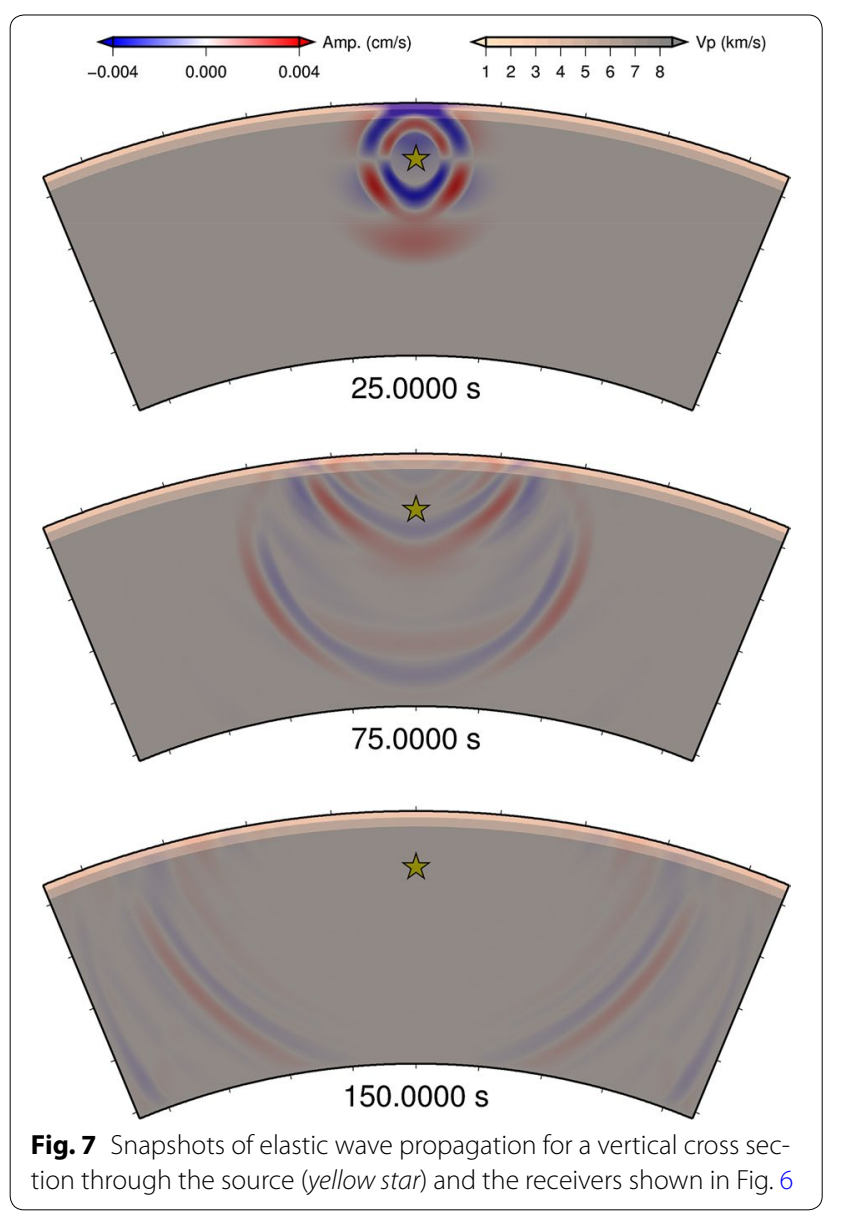

\section{Simulation for an Earth section around Japan}

We show the target area (around Japan) for the next simulation in Fig. 8. This simulation area is much larger than that for usual local strong-motion simulations which can often be carried out with Cartesian FDTD. The right panel of Fig. 8 displays the computational area moved around the equator by the equatorial-to-ecliptic coordinate transformation. The computational domain is discretized with cells of $0.05^{\circ} \times 0.05^{\circ} \times 0.5 \mathrm{~km}$. The total grid size is then $1601 \times 2401 \times 401$. The time step is $0.025 \mathrm{~s}$. The structural model is the $3-\mathrm{D}$ model that Nakamura et al. (2015) used for a strong-motion simulation of a real moderate earthquake, one of aftershocks of the 1995 Kobe earthquake, for land and ocean-bottom seismic networks. This model incorporates realistic 3-D heterogeneous velocity, density, and anelastic attenuation structures including a seawater layer, the seafloor and land surface topography, sediment layers and crust and upper mantle for the continental and the oceanic plates. Anelastic attenuation could be implemented by using a viscoelastic formulation (e.g., Blanch et al. 1995; JafarGandomi and Takenaka 2013) instead of the elastic one, and it is straightforward to apply the quasi-Cartesian scheme to the viscoelastic formulation. We here demonstrate a simulation of seismic wave propagation using a 3-D structural model with anelastic attenuation.

We set a virtual point source in the Hyuga-nada area in the southwestern Japan for the simulation. We assume the source depth of $21 \mathrm{~km}$, which is in the oceanic crust

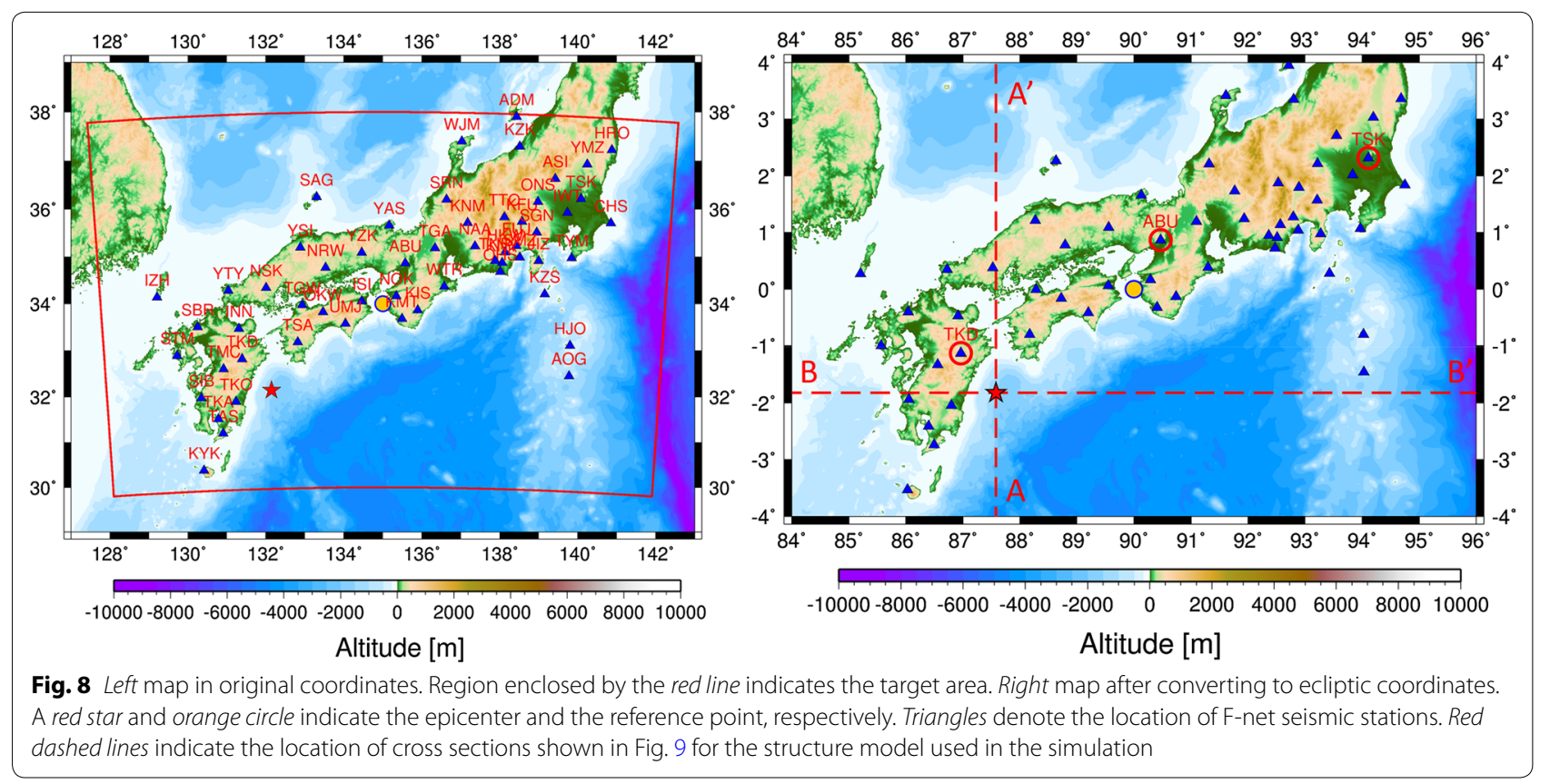


of the Philippine Sea slab (Fig. 9), and a thrust-type event (strike $=\mathrm{N} 22.4^{\circ} \mathrm{E}$, dip $=79^{\circ}$, rake $=76^{\circ}$ ) with seismic moment of $5.33 \times 10^{17} \mathrm{Nm}\left(M_{\mathrm{W}} 5.8\right)$ whose source time function is a bell-shaped pulse with width of $2.5 \mathrm{~s}$. We output the seismograms at F-net stations within the simulation area, which are operated by the National Research Institute for Earth Science and Disaster Resilience (Okada et al. 2004).

Figure 10 shows the synthetic seismograms at three stations (TKD, ABU, and TSK) marked in the right panel of Fig. 8, for the spherical Earth (quasi-Cartesian FDTD) and the corresponding flat Earth (Cartesian FDTD). The epicentral distances of the three stations are 103.5, 438.7, and $859.3 \mathrm{~km}$ for TKD, ABU, and TSK, respectively. The waveforms have been bandpass-filtered in the period range 10-20 s. At station TKD nearest to the epicenter, the traces of all components from the quasi-Cartesian FDTD are identical to those from the Cartesian FDTD within the line thickness, which means the contribution of the additional terms in Eqs. (8) and (9) is tiny at this distance. At stations ABU and TSK, the discrepancy between the traces for the 3-D spherical and the 3-D flat Earth models is visible, and at TSK in particular the difference between them looks clear around $200 \mathrm{~s}$ (S phase). Figure 11 displays the synthetic seismograms from all stations shown in Fig. 8 along to the epicentral distances. The difference between waveforms obtained by the two FDTDs is clear beyond about $500 \mathrm{~km}$ in this case.

\section{Conclusions}

We have described a simple and efficient finite-difference scheme, called the quasi-Cartesian FDTD to calculate seismic wave propagation for a sub-global (regional or larger) scale model represented by a partial spherical shell of a 3-D heterogeneous global Earth structure. This scheme solves the elastodynamic equation in the quasi-Cartesian coordinate system similar to a local Cartesian system. We have demonstrated accuracy and validity of the present scheme and the feasibility to apply it to real large simulations via two numerical examples. The present scheme can be easily implemented using a 3-D Cartesian FDTD code of

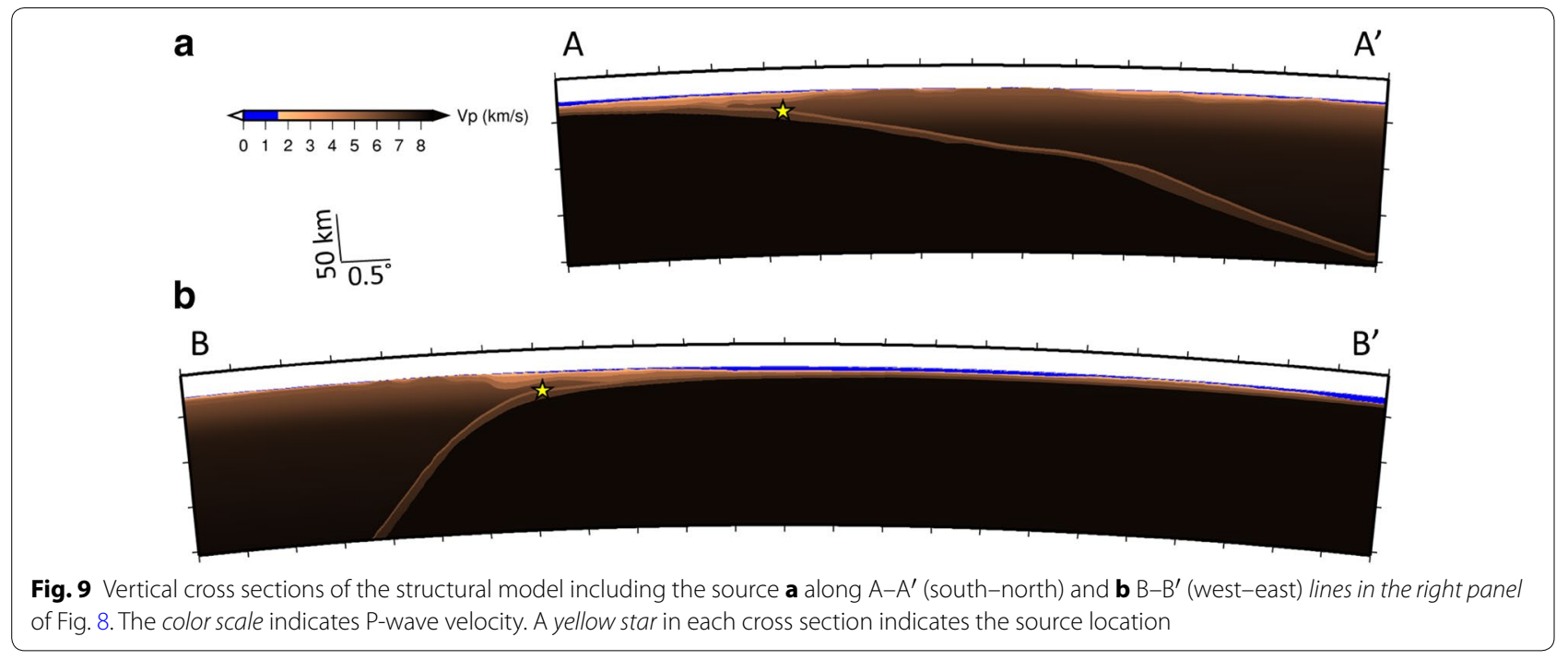


TKD
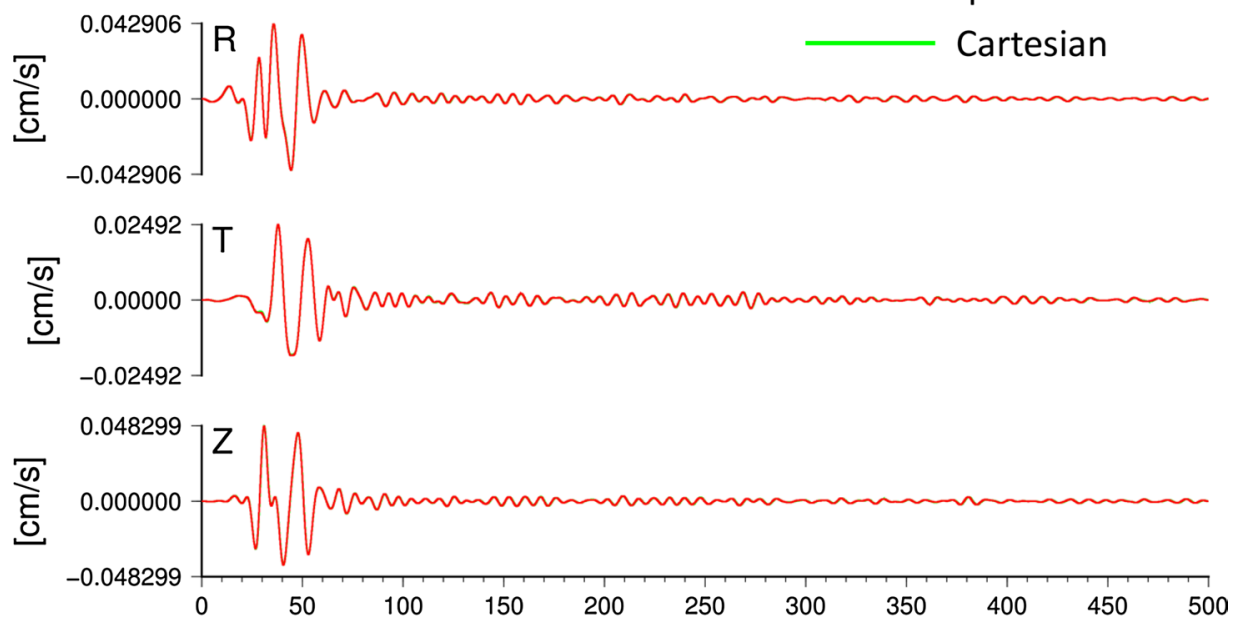

[s]

$\mathrm{ABU}$
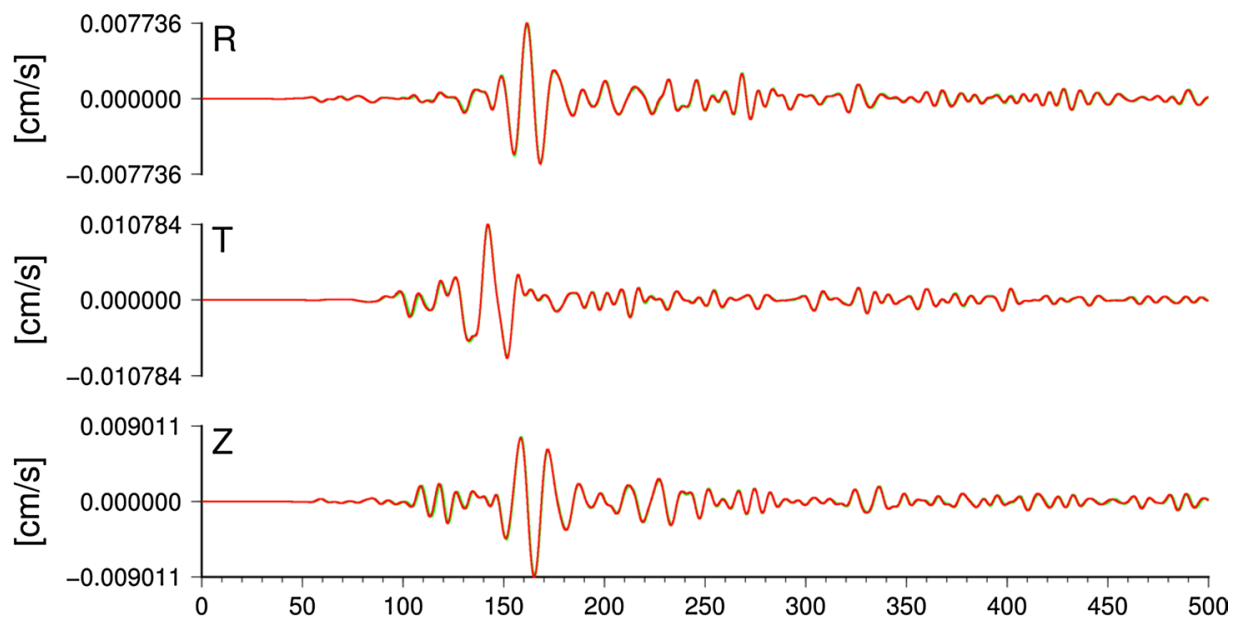

[s]
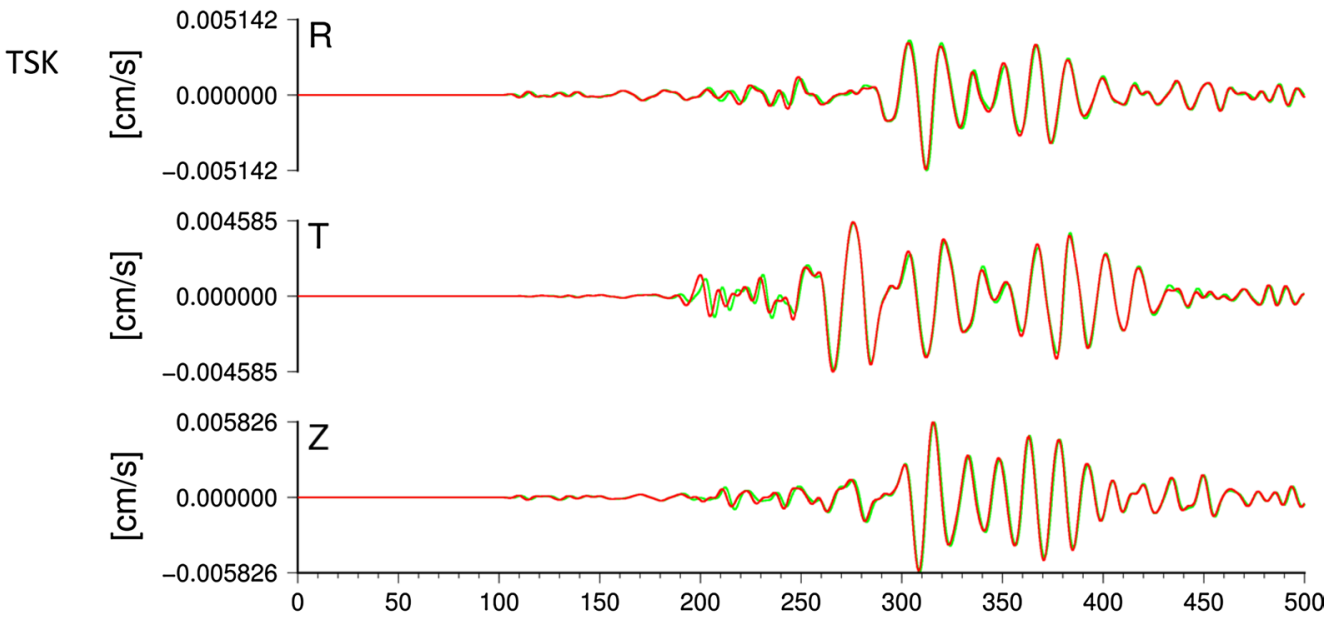

[s]

Fig. 10 Comparison between synthetic waveforms by the quasi-Cartesian (red) and the Cartesian (green) FDTDs at three stations marked in the right panel of Fig. 8 

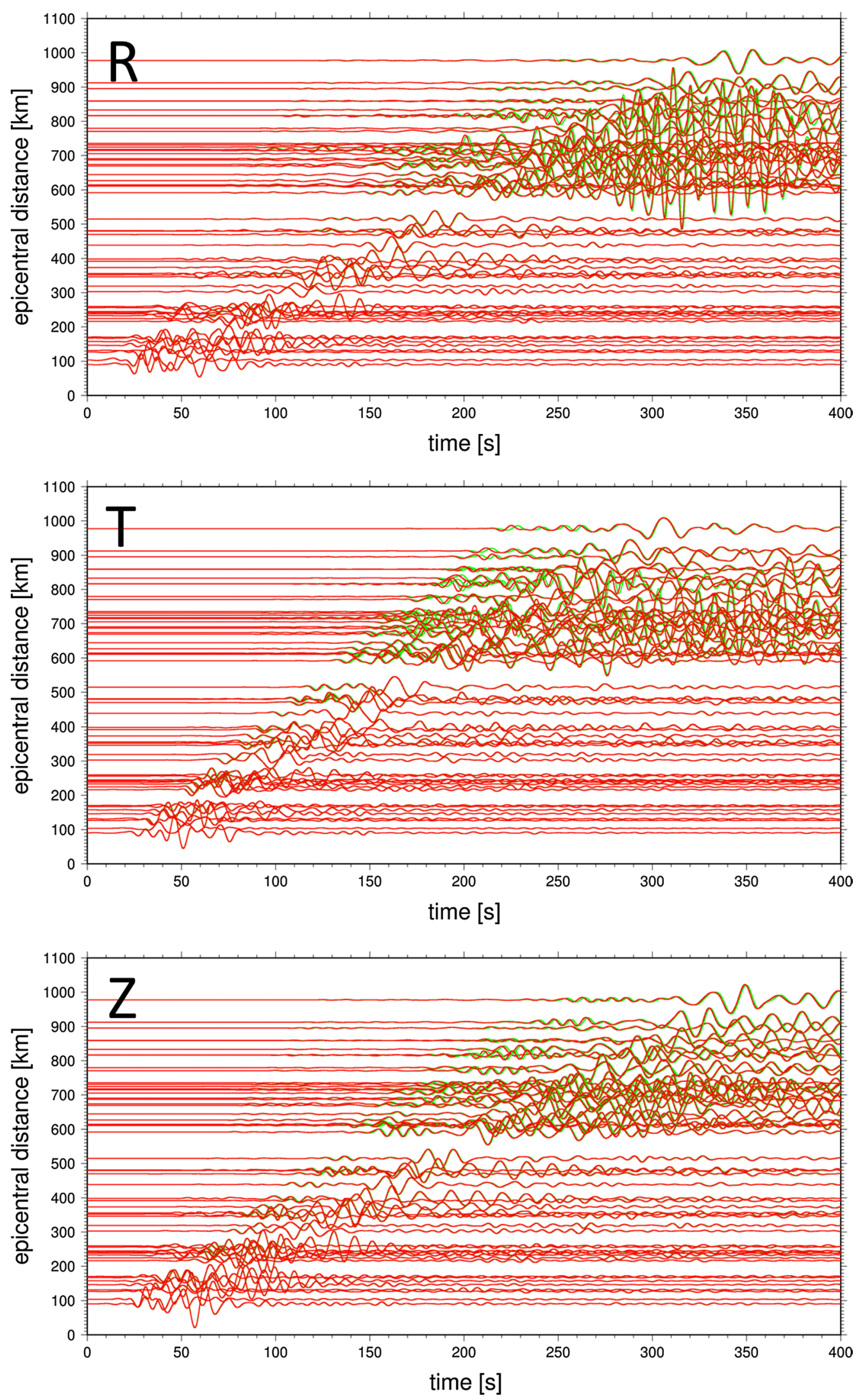
(See figure on previous page.)

Fig. 11 Comparison between synthetic waveforms from the quasi-Cartesian (red) and the Cartesian (green) FDTDs at all stations in the target area shown in the left panel of Fig. 8. Upper, middle, and lower panels show the waveforms in the radial, transverse, and vertical components, respectively. These waveforms are set along to the epicentral distances. The amplitude of each trace has been multiplied by the epicentral distance. Each trace has been bandpass-filtered in the period range 10-20 s

local-scale modeling by changing a very small part of the code. The quasi-Cartesian approach may be able to open a window for multiscale modeling ranging from global to local scales.

\section{Abbreviations}

3-D: three-dimensional; FDM: finite-difference method; FDTD: (staggered-grid) finite-difference method in time domain; PML: perfectly matched layer(s).

\section{Authors' contributions}

HT developed the quasi-Cartesian finite-difference scheme for sub-global seismic wave propagation modeling. HT, MK, GT, and TN participated in developing the simulation codes and carrying out the numerical tests. TO participated in the study design. HT and MK drafted the manuscript. All authors read and approved the final manuscript.

\section{Author details}

${ }^{1}$ Department of Earth Sciences, Okayama University, 3-1-1 Tsushima-Naka, Kita-ku, Okayama 700-8530, Japan. ${ }^{2}$ Department of Geophysics, Tohoku University, 6-6 Aza-Aoba, Aramaki, Aoba-ku, Sendai 980-8578, Japan. ${ }^{3}$ Earthquake and Tsunami Research Division, National Research Institute for Earth Science and Disaster Resilience, 3-1 Tennodai, Tsukuba, Ibaraki 305-0006, Japan. ${ }^{4}$ Department of Earth and Planetary Sciences, Tokyo Institute of Technology, 2-12-1 Ookayama, Meguro, Tokyo 152-8551, Japan.

\section{Acknowledgements}

We are grateful to the editor, Prof. Kimiyuki Asano, and the two anonymous reviewers who provided us with constructive comments and suggestions that have improved this paper. We used the Generic Mapping Tools (Wessel, P. \& Smith, W. H. F. EOS Trans. AGU 79, 579, 1998) for drawing some figures. This study is partially supported by KAKENHI (26282105) and JHPCN (jh160029-NAH)

\section{Competing interests}

The authors declare that they have no competing interests.

\section{Publisher's Note}

Springer Nature remains neutral with regard to jurisdictional claims in published maps and institutional affiliations.

Received: 30 December 2016 Accepted: 2 May 2017

Published online: 12 May 2017

\section{References}

Aki K, Richards PG (2002) Quantitative seismology. University Science Book, Sausalito

Blanch JO, Robertsson JOA, Symes WW (1995) Modeling of a constant Q: methodology and algorithm for an efficient and optimally inexpensive viscoelastic technique. Geophysics 60:176-184

Borisov D, Singh SC, Fuji N (2015) An efficient method of 3-D elastic full waveform inversion using a finite-difference injection method for time-lapse imaging. Geophys J Int 202:1908-1922

Fornberg B (1988) Generation of finite difference formulas on arbitrarily spaced grids. Math Comp 51:699-706

Garcia RF, Gagnepain-Beyneix J, Chevrot S, Lognonné P (2011) Very preliminary reference Moon model. Phys Earth Planet Int 188:96-113
Graves RW (1996) Simulating seismic wave propagation in 3-D elastic media using staggered-grid finite differences. Bull Seismol Soc Am 86:1091-1106

Hayashida T, Takenaka H, Okamoto T (1999) Development of 2D and 3D codes of the velocity-stress staggered-grid finite-difference method for modeling seismic wave propagation. Sci Rep Dept Earth Planet Sci Kyushu Univ 20:99-110 (in Japanese with English abstract)

Igel H, Gudmundsson O (1997) Frequency-dependent effects on travel times and waveforms of long-period S and SS waves. Phys Earth Planet Int 104:229-246

Igel H, Nissen-Meyer T, Jahnke G (2002) Wave propagation in 3D spherical sections: effects of subduction zones. Phys Earth Planet Int 132:219-234

JafarGandomi A, Takenaka H (2013) FDTD3C - a FORTRAN program to model multi-component seismic waves for vertically heterogeneous attenuative media. Comput Geosci 51:314-323. doi:10.1016/j.cageo.2012.07.022

Moczo P, Bystrický E, Kristek J, Carcione JM, Bouchon M (1997) Hybrid modeling of P-SV seismic motion at inhomogeneous viscoelastic topographic structures. Bull Seismol Soc Am 87:1305-1323

Moczo P, Kristek J, Gális M (2014) The finite-difference modelling of earthquake motions. Cambridge University Press, Cambridge

Monteiller V, Chevrot S, Komatitsch D, Fuji N (2013) A hybrid method to compute short-period synthetic seismograms of teleseismic body waves in a 3-D regional model. Geophys J Int 192:230-247

Nakamura T, Takenaka H, Okamoto T, Kaneda Y (2012) FDM simulation of seismic-wave propagation for an aftershock of the 2009 Suruga bay earthquake: effects of ocean-bottom topography and seawater layer. Bull Seismol Soc Am 102:2420-2435. doi:10.1785/0120110356

Nakamura T, Takenaka H, Okamoto T, Ohori M, Tsuboi S (2015) Long-period ocean-bottom motions in the source areas of large subduction earthquakes. Sci Rep 5:Article number: 16648. doi:10.1038/srep16648

Okada Y, Kasahara K, Hori S, Obara K, Sekiguchi S, Fujiwara H, Yamamoto A (2004) Recent progress of seismic observation networks in JapanHi-net, F-net, K-NET and KiK-net. Earth Planets Space 56:xv-xxviii. doi:10.1186/BF03353076

Opršal I, Matyska C, Irikura K (2009) The source-box wave propagation hybrid methods: general formulation and implementation. Geophys J Int 176:555-564

Pitarka A (1999) 3D elastic finite-difference modeling of seismic motion using staggered grids with nonuniform spacing. Bull Seismol Soc Am 89:54-68

Robertsson JOA, Chapman CH (2000) An efficient method for calculating finitedifference seismograms after model alterations. Geophysics 65:907-918

Takenaka H, Nakamura T, Okamoto T, Kaneda Y (2009) A unified approach implementing land and ocean-bottom topographies in the staggeredgrid finite-difference method for seismic wave modeling. In: Proceedings of the 9th SEGJ (Society of Exploration Geophysicists of Japan) international symposium, Sapporo, Japan, 12-14 October 2009: 1-4. doi:10.1190/segj092009-001.13

Thomas Ch, Igel H, Weber M, Scherbaum F (2000) Acoustic simulation of P-wave propagation in a heterogeneous spherical earth: numerical method and application to precursor waves to PKPdf. Geophys J Int 141:307-320. doi:10.1046/j.1365-246x.2000.00079.x

Toyokuni G, Takenaka H (2006) FDM computation of seismic wavefield for an axisymmetric earth with a moment tensor source. Earth Planets Space 58:e29-e32

Toyokuni G, Takenaka H (2012) Accurate and efficient modeling of global seismic wave propagation for an attenuative Earth model including the center. Phys Earth Planet Int 200-201:45-55. doi:10.1016/j. pepi.2012.03.010

Toyokuni G, Takenaka H, Wang Y, Kennett BLN (2005) Quasi-spherical approach for seismic wave modeling in a 2-D slice of a global Earth model with lateral heterogeneity. Geophys Res Lett 32:L09305. doi:10.1029/200 4GL022180 
Toyokuni G, Takenaka H, Kanao M (2012) Quasi-axisymmetric finite-difference method for realistic modeling of regional and global seismic wavefield —review and application. In: Kanao M (ed) Seismic wavesresearch and analysis. InTech Publisher, Rijeka, Croatia, pp 85-112. doi:10.5772/32422

Wen L, Helmberger DV (1998) A two-dimensional P-SV hybrid method and its application to modeling localized structures near the core-mantle boundary. J Geophys Res 103:17901-17918
Yoshimura C, Bielak J, Hisada Y, Fernández A (2003) Domain reduction method for three-dimensional earthquake modeling in localized regions, part II: verification and applications. Bull Seismol Soc Am 93:825-841

\section{Submit your manuscript to a SpringerOpen ${ }^{\circ}$ journal and benefit from:}

- Convenient online submission

\section{- Rigorous peer review}

- Immediate publication on acceptance

- Open access: articles freely available online

- High visibility within the field

- Retaining the copyright to your article 